\title{
Defects detection by infrared thermography with a new microwave excitation system
}

\author{
Sam Ang Keo a , Franck Brachelet, Didier Defer and Florin Breaban \\ Laboratoire Génie Civil et Géo-Environnement (LGCgE), PRES Lille Nord de France, Faculté des Sciences \\ Appliquées, Technoparc Futura, 62400 Béthune, France
}

Received 7 January 2014, Accepted 15 May 2014

\begin{abstract}
This study presents a NDT method using infrared thermography associated with a microwave excitation. The advantages of such stimulation lie in the volumic absorption of incoming waves which lead to a greater sounded depth. This method is applied to two types of samples. The first is a concrete slab reinforced with CFRP on which a bonding failure is inserted and the second is a wooden plate on which a metallic insert is placed on the back face. The device generating the microwaves is made of a commercial magnetron associated with a pyramidal horn antenna. An infrared camera is placed on the same side as the stimulated surface and thermograms are recorded at regular intervals. The whole assembly is placed in a protective room against high frequencies. The incident power density leads to heating of less than $1{ }^{\circ} \mathrm{C}$ of the surface of the samples. The thermograms show a higher temperature rise in front of the defect area. The non-uniformity of the beam, leads us to treat the thermograms with an algorithm of contrast. These first results show the interest of the microwave excitation to detect defects deeper than in the case of surface excitation.
\end{abstract}

Key words: NDT / infrared thermography / microwave / CFRP / wood

\section{Introduction}

In Civil Engineering, we are witnessing the development of many inspection methods. They are intended to evaluate the conditions of structures by providing information about the internal structures or the status of the materials. The techniques based on infrared thermography are promising [1-11]. They have high performance and results in the observation of thermal images, which seems more accessible $[11,12]$. In mechanical engineering field, non-destructive testing by infrared thermography has been the subject of numerous studies. The applications in mechanical assemblies control or in studies of composite materials have been implemented and well mastered since long time ago [13]. The methods that have been developed are based on the analysis of the temperature response of a surface of a structure correlated with a controlled thermal solicitation. Many signal processing techniques have been used to extract the information of the thermograms [11]. With the appearance on the market of measuring equipments more flexible to use and suitable for outdoor uses, the researchers are trying to transpose the existing thermography methods to civil engineering.

${ }^{a}$ Corresponding author: anggci@yahoo.com
The transition to these applications is done with different constraints mainly related to the size and the material nature of the detected structures. It still remains the problem of implementing the appropriate thermal solicitations.

In this study, a microwave excitation system enables to heat the bigger surface samples. It replies to the needs for the applications in engineering. The system gives a transmission of microwave beam onto the samples by direct diffusion without the need of putting them into the cavity of an oven as the existing methods [14-16]; this solution is an economical one.

\section{Microwave excitation system}

The generation of the electromagnetic waves is carried out by using a commercial magnetron whose power is $800 \mathrm{~W}$ at a frequency of $2.45 \mathrm{GHz}$. The generator was adapted to a horn antenna with an opening of $40^{\circ}$ serving to guide the microwave beam onto the tested samples. The control part was kept so that the power and duration of radiation were adjusted. The entire system is assembled on a tripod adjustable in azimuth and elevation (Fig. 1). The radiation pattern of the antenna is shown in Figure 3; 


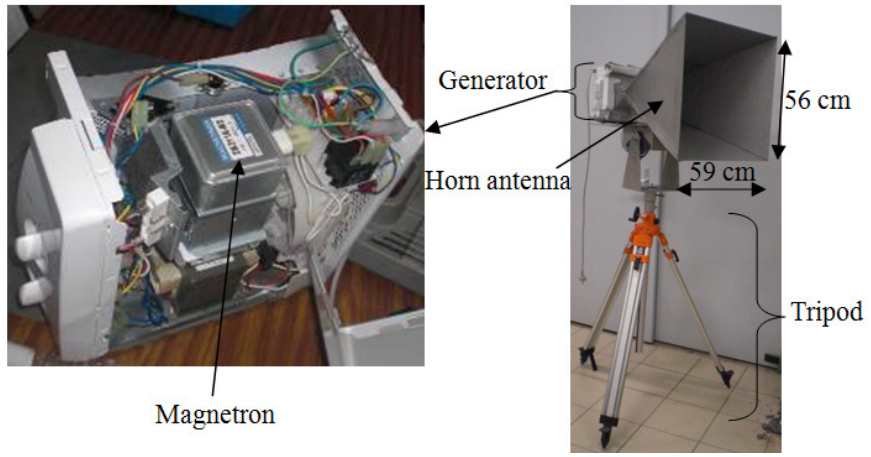

Fig. 1. Microwave excitation system.

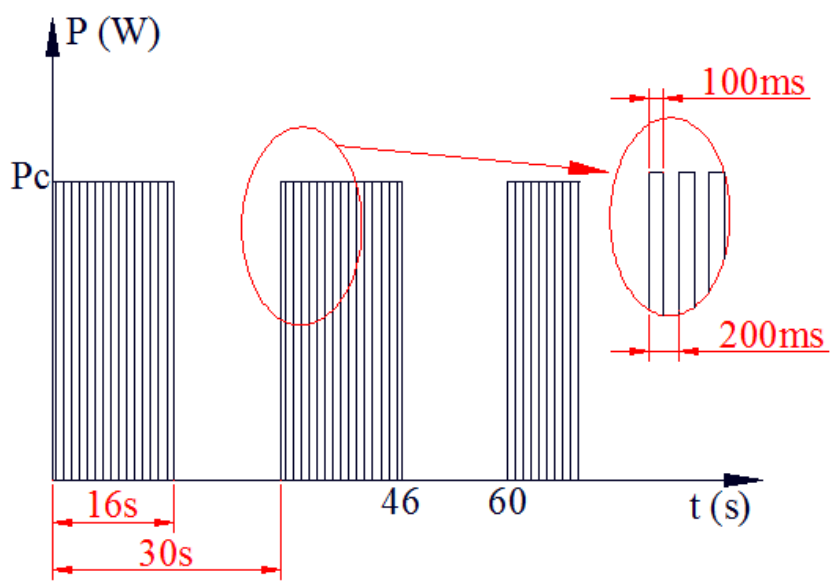

Fig. 2. Microwave signals.

it has a gain of $21.67 \mathrm{~dB}$ determined in its main directions by the equation below $[17,18]$ :

$$
G_{\mathrm{dB}}=10 \log _{10}\left[\left(4 \pi / \lambda^{2}\right) \varepsilon_{\mathrm{ap}} A B\right]
$$

where

$\lambda$ : wavelength $(12 \mathrm{~cm}$ at $2.45 \mathrm{GHz})$;

$A, B$ : aperture dimensions $(A=59 \mathrm{~cm}, B=56 \mathrm{~cm})$;

$\varepsilon_{\text {ap }}$ : aperture efficiency ( 0.51 generally).

The signals controlled from the magnetron are used as references to know the waveform of the power sent onto the samples. The envelope of the waveform can be regarded as a pulsed train having variable width according to the transmitted power. Figure 2 shows the microwave signals detected by a sensor. At the average power of $360 \mathrm{~W}$, the signals form as a pulsed train with a period of $30 \mathrm{~s}$ and a pulse duration of $16 \mathrm{~s}$. In each slot of the train, there is a modulation period of $200 \mathrm{~ms}$ and pulse duration of $100 \mathrm{~ms}$.

The absorption of microwave was generated by the water molecules in the tested materials. The measurement of the microwave signal in the far field $(25 \mathrm{~m}$ from the antenna) in the vertical and horizontal directions enabled to obtain the radiation pattern in a polar axis system (Fig. 3). The horizontal lobe represents the main lobe of the microwave radiation in the magnetic plane (H-plane). The vertical lobe is the main lobe of the microwave beam radiated in the electric plane (E-plane).

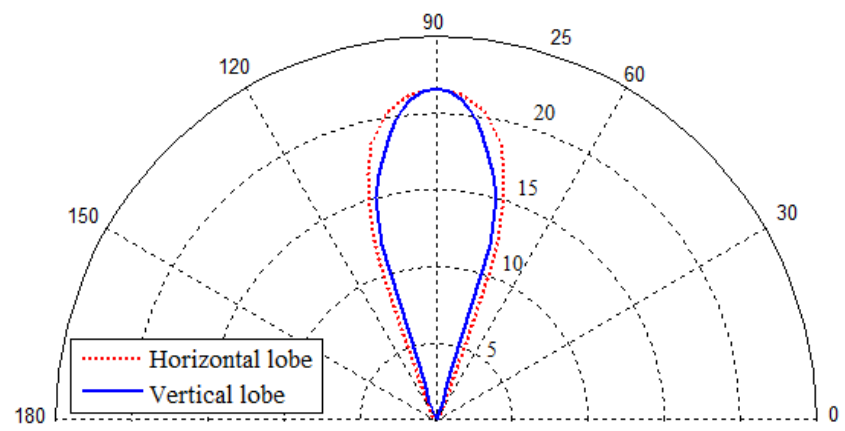

Fig. 3. Radiation pattern (principal lobes).

\section{Samples}

Two types of material were tested: a concrete plate reinforced by carbon fiber reinforced polymer and a wooden plate with a metallic element placed on its rear face. For each type, two samples were prepared, one without defect and another one with a controlled defect.

On a concrete plate $0.4 \mathrm{~m} \times 0.4 \mathrm{~m}$ of dimensions and $4.5 \mathrm{~cm}$ of thickness, a composite material of carbon fiber of $20 \mathrm{~cm}$ width and $1 \mathrm{~mm}$ thick is fastened with the aid of Sikadur adhesive layer about $1 \mathrm{~mm}$ of thickness (Fig. 4a). The absence of adhesive $10 \mathrm{~cm} \times 10 \mathrm{~cm}$ at the middle of another sample represents a bonding failure. The other type, there are two wooden plates $0.9 \mathrm{~m} \times 1.2 \mathrm{~m}$ and $1 \mathrm{~cm}$ of thickness. A circular metal element having a thickness of $4 \mathrm{~mm}$ and $5 \mathrm{~cm}$ of diameter was fixed against the rear face of one of these plates (Fig. 4b).

\section{Experimental campaigns}

\subsection{Protection against microwave radiation}

In order to respect to the safety instructions and the protection against high frequency electromagnetic fields, a metal grill was used to prevent microwave leakage outside the surrounding wall $[19,20]$. A finely woven wire mesh (1 mm mesh) of HEG10 type was used as a shield. This grill can mitigate $30 \mathrm{~dB}$ the microwaves from magnetron at the frequency of $2.45 \mathrm{GHz}$, which corresponds to $99.9 \%$ loss. The protective fence is shown in Figure 5.

The cutting wire mesh was attached to each metallic barrier to form many shields. The screen dividing the area of acquisitions (computer synchronized to the infrared camera) and the microwave system with detector is made by superimposing two grill layers to assure a more effective protection for operators.

Safety standards limit the exposure of the operators to a power density less than $5 \mathrm{~mW} / \mathrm{cm}^{2}$ [21]. After installing the protection system, the measurement of the microwaves passing through the shield (especially the double layers-screen because it protects the operators from microwaves radiation during the infrared thermography tests) was carried out. A digital microwave detector, placed in the area 1, measured the microwaves getting 


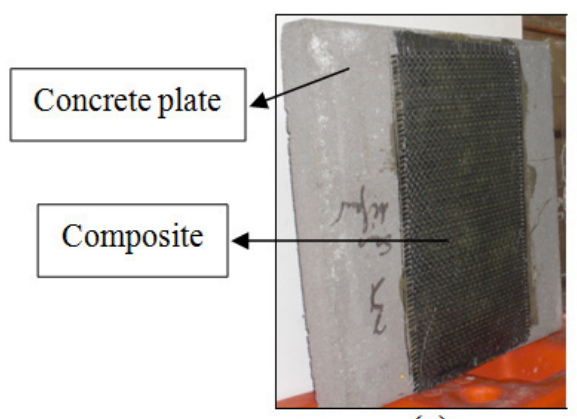

(a)

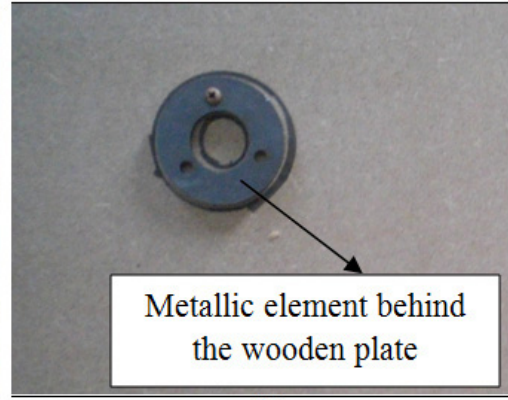

(b)

Fig. 4. Samples with defect, (a) composites, (b) wood.
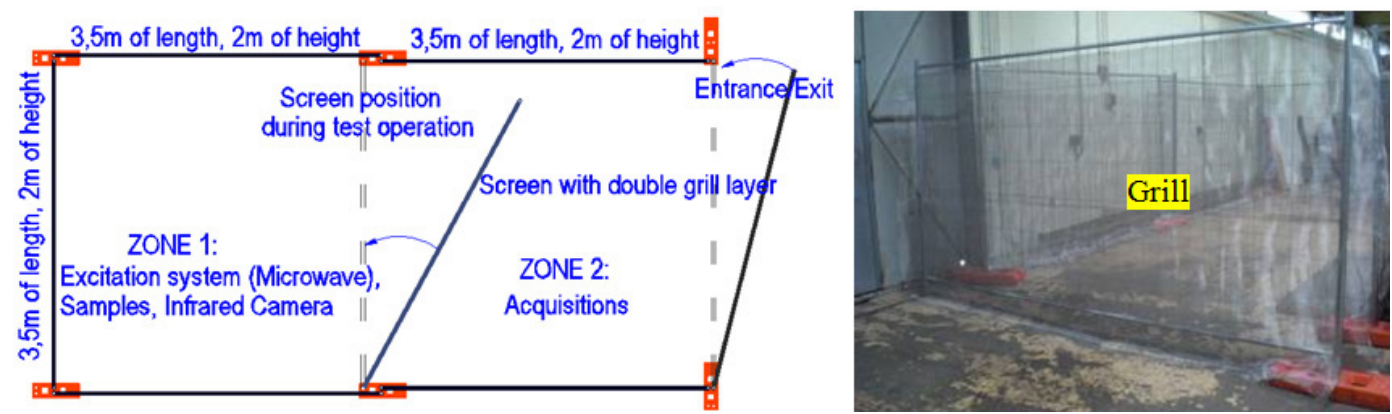

Fig. 5. Protective enclosure.

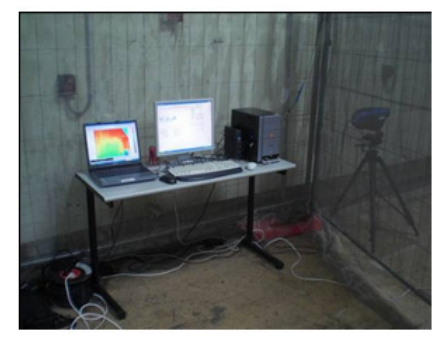

(a)

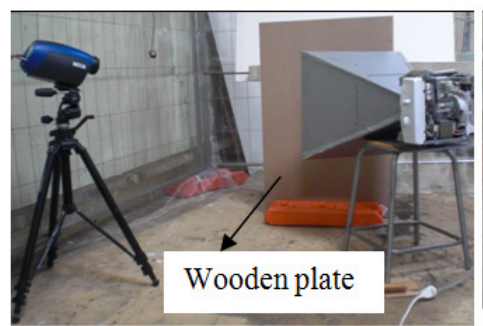

(b)

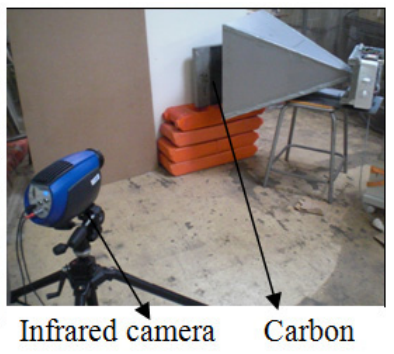

(c)

Fig. 6. Experimental test set-up, (a) acquisition part, (b) excitation part with wooden samples, (c) excitation part with carbon samples.

through the screen to the area 2 (Fig. 5) with the digital microwave sensor. When the value given by the detector exceeded the security value, one more mesh layer may be added to decrease the passing microwaves. The power density of the microwave passing through the shield was always less than $5 \mathrm{~mW} / \mathrm{cm}^{2}$ during the tests.

\subsection{Test set-up}

The position of the samples was marked, at $20 \mathrm{~cm}$ from the antenna aperture. An infrared camera sensitive to medium waves in range of $3-5 \mu \mathrm{m}$, with a $320 \times$ 256 matrix detector in InSb (Indium Antimonide), was placed at $1.5 \mathrm{~m}$ from the sample so as to detect the whole area heated by the microwave beam from the antenna (Figs. 6b and 6c). The assembly was placed in the protective enclosure (Fig. 5). The thermograms were recorded at regular intervals by a computer placed in acquisition area
(Fig. 6a), using the ALTAIR software. System startup microwave is controlled from the acquisition area. The microwave system start-up was controlled from the acquisition area too. The specimen was heated with an average power of $360 \mathrm{~W}$. After recording the thermograms, another sample (of the same type of material) was then placed in the same place (without moving the camera and antenna) to repeat the procedure.

\section{Results}

\subsection{Principle of data analysis}

The method used to analyze the thermogram series is based on a contrast algorithm. The non-uniformity of the beam produced an inhomogeneous heat generated in the sample and may lead to misinterpretations. The amplitude of this non-uniformity also depends on the nature of 


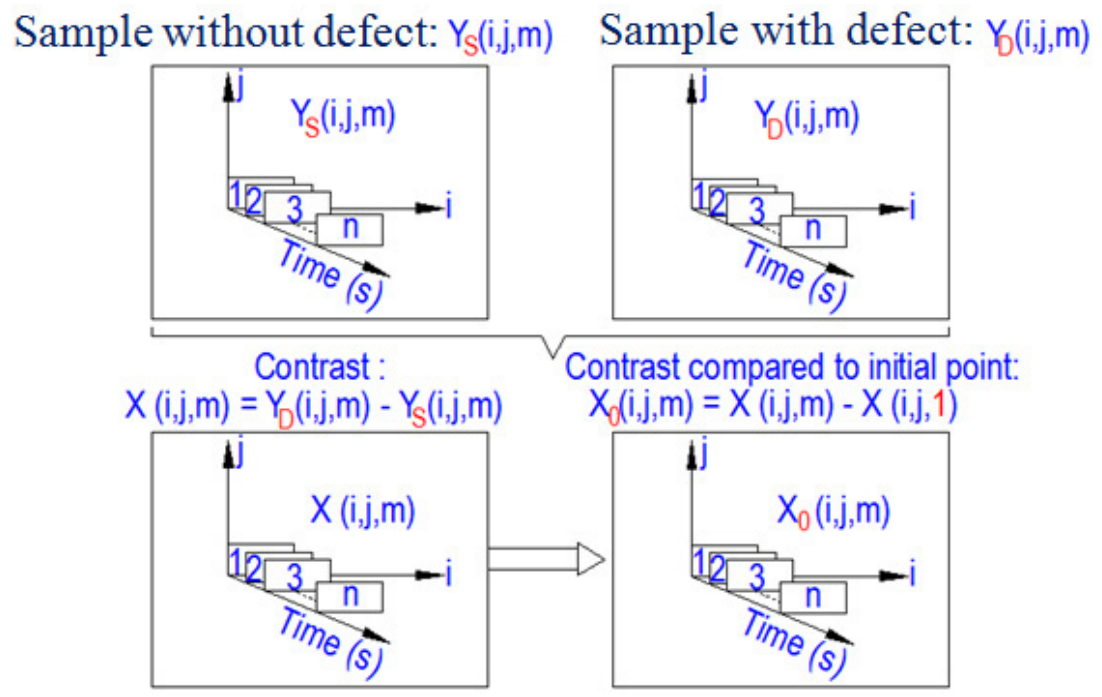

Fig. 7. Schema of thermogram analysis principle.
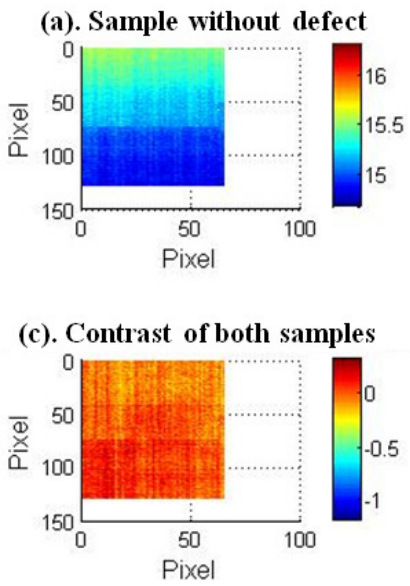

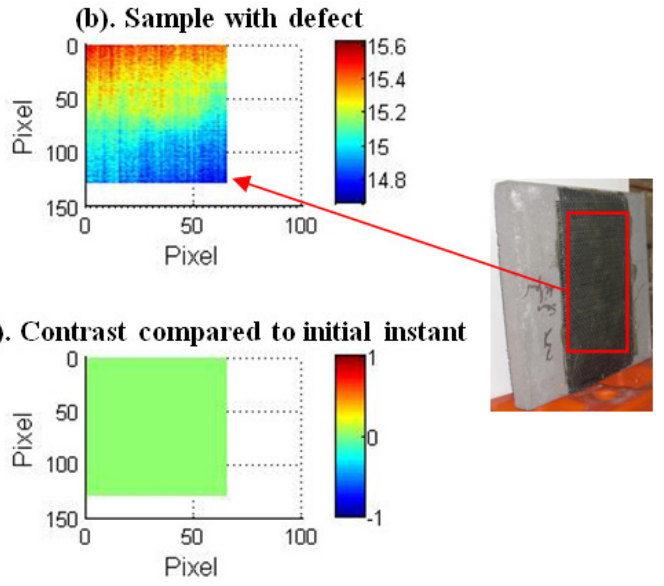

Fig. 8. Thermograms of composite samples at the initial instant.

the inspected materials. Thus, the tests were conducted in two stages. Considering a point $Y(i, j)$ on the surface of the tested samples, the difference $(X)$ was carried out at the same instants between the temporal evolution of the series with defect $\left(Y_{\mathrm{D}}\right)$ and without defect $\left(Y_{\mathrm{S}}\right)$. This approach can reduce the effect of the non-uniformity of the solicitation. The initial temperature value was then subtracted from the contrast sequence, which allowed to consider only the temperature rise $\left(X_{0}\right)$ in case the initial temperatures of both samples would not be identical. The algorithm was applied to each point comprising the thermograms.

The principle of the interpretation is shown in Figure 7.

\subsection{Results and discussions}

\subsubsection{Composites from carbon fiber}

Figure 8 shows the application of the previous algorithm to all the points of the sample surface at the initial time. Although there is a temperature gradient in the initial instant for both samples with defect and without defect (Figs. 8a and 8b), Figure 8c doesn't show any area representing a defect.

The contrast function enables to get an instant at which Figure 10d presents a maximal contrast (Fig. 9).

Figure 10 shows the thermograms obtained at the instant of $100 \mathrm{~s}$. The contrast algorithm obviously shows the defected area corresponding to the absence of the adhesive under the carbon fiber tissue.

The sample was been heating for $150 \mathrm{~s}$. The two curves (Fig. 11) show an increase in the average temperature of the two areas. The curves are modulated by the operating phases of the microwave. At the end of the heating stage, it occurs a phase of rapid relaxation of the temperature tending to return to its initial point. The two points on the sample without defect have an identical increase of $0.4^{\circ} \mathrm{C}$ during the heating stage.

The second series of measurements on the sample with defect shows a difference of temperature changes, $0.8^{\circ} \mathrm{C}$ for the defected area and $0.5{ }^{\circ} \mathrm{C}$ for the healthy area. 


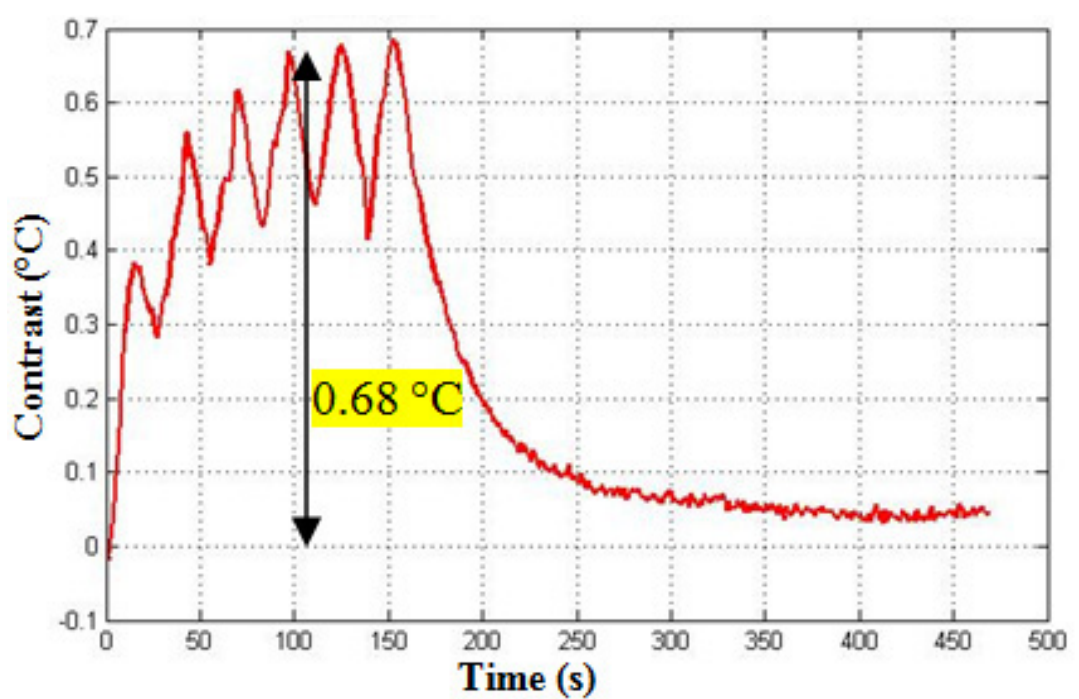

Fig. 9. Evolution of thermal contrast of composite samples.

(a). Sample without defect

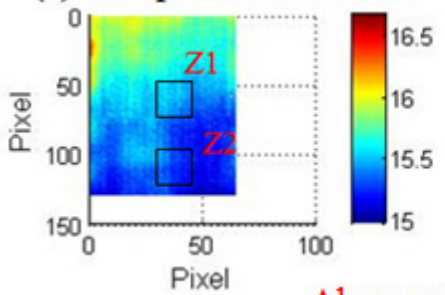

Absence of adhesive (b). Sample with defect

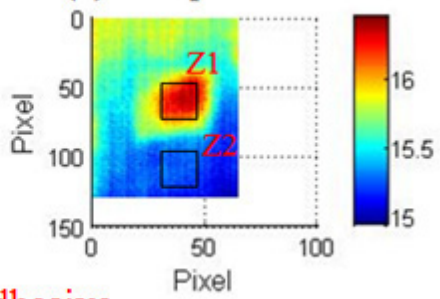

(d). Contrast compared to initial instant

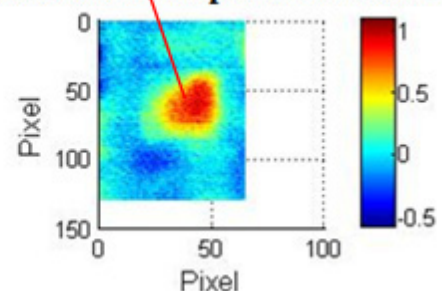

Fig. 10. Thermograms of the composite samples at the instant of $100 \mathrm{~s}$.

(a). Sample without defect

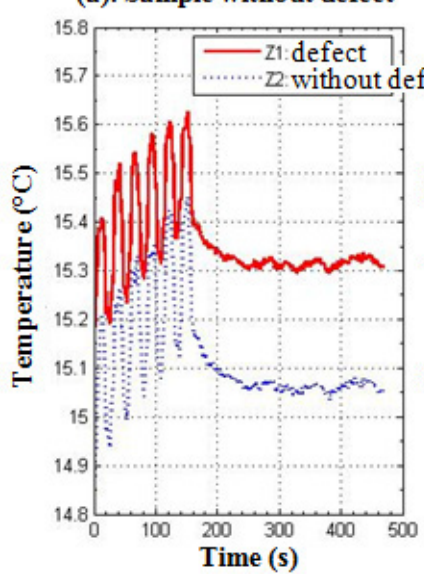

(b). Sample with defect

(c). Contrast between both samples
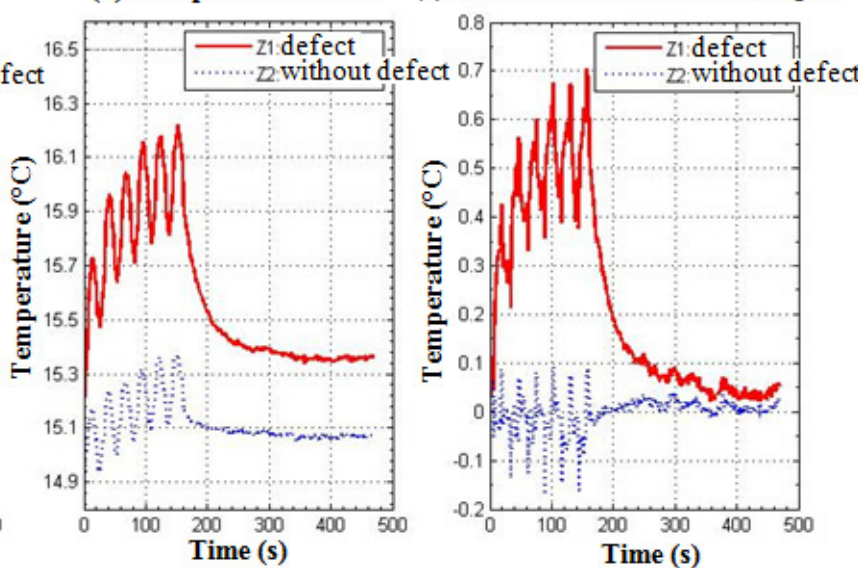

Fig. 11. Evolution of the surface temperatures of the composite samples. 


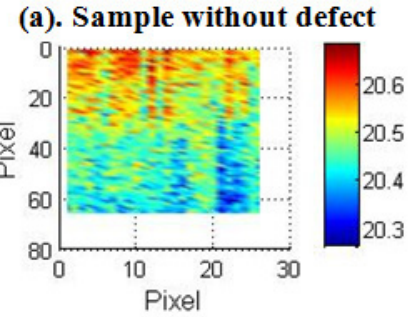

(c). Contrast of both samples

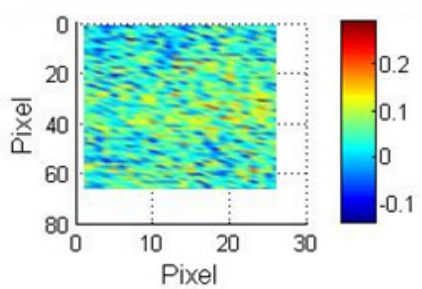

(b). Sample with defect

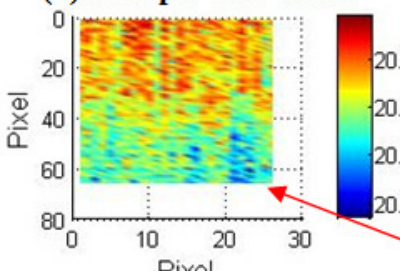

(d). Contrast compared to initial instant

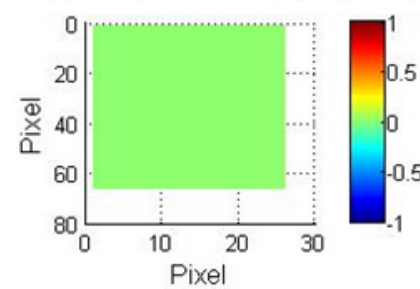

Fig. 12. Thermograms of the wooden plate at the initial instant.

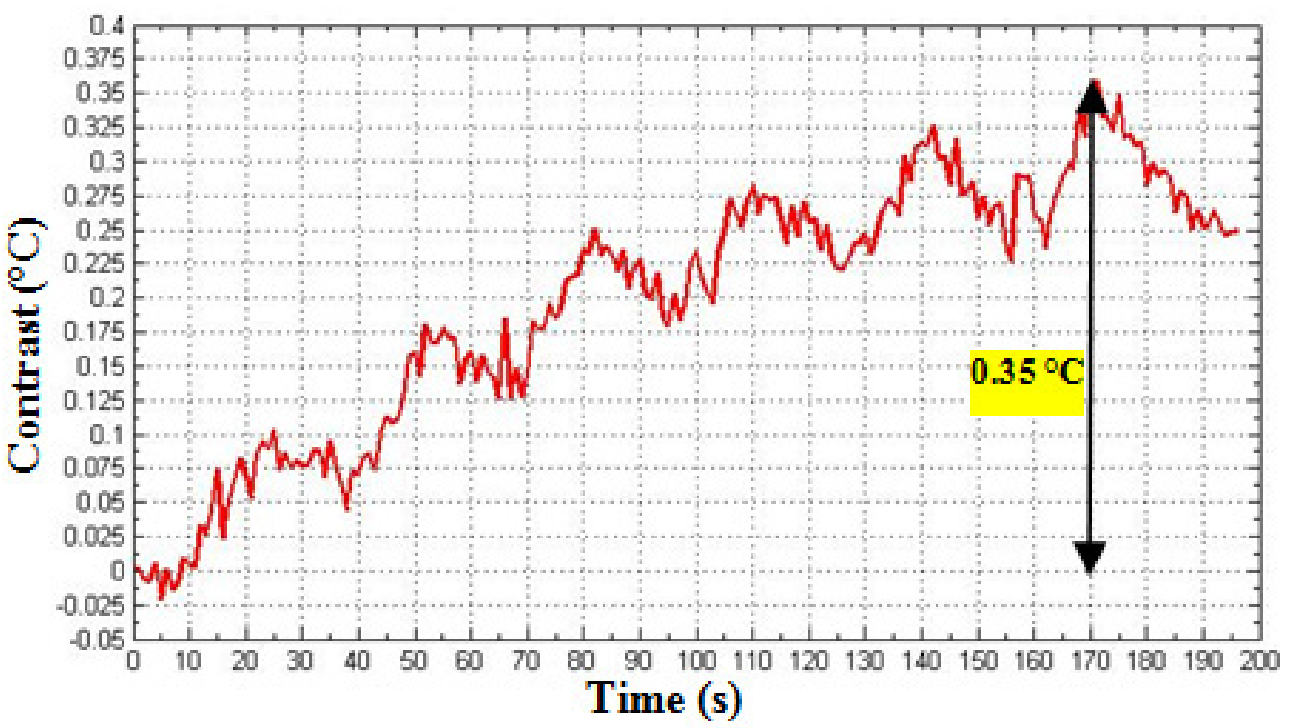

Fig. 13. Evolution of the thermal contrast of the wooden plate samples.

The difference between these curves shows (Fig. 11c) that there is only a very small temperature difference for the healthy area while the defected area shows a wide gap due to the defect up to $0.7^{\circ} \mathrm{C}$.

\subsubsection{Wood}

The wooden plate sample was been heating for $180 \mathrm{~s}$, the thermograms below were taken at the initial instant. The thermograms don't show the presence of a defect, but only the presence of a temperature gradient between the top and bottom of the plate.

The contrast function below shows that the maximal contrast is obtained at the instant of $170 \mathrm{~s}$ (Fig. 12).

The first test on a wooden plate without the metallic element shows a moderate increase in temperature $\left(0.7^{\circ} \mathrm{C}\right)$. The wood is heated under the effect of the microwaves on the residual water contained in the fibers. The second test was conducted by placing a metallic element against the rear face of the sample allowing the microwaves to be reflected there. These reflected waves were then absorbed again by the water contained in the wood and led to a rise in temperature greater than other areas (without metallic element).

The thermograms obtained at the instant of $170 \mathrm{~s}$ (Fig. 14) show the presence of the metallic element behind the wooden plate. The difference in average temperature between the area with the metallic element (defected area) and the area without metallic element (area without defect) is about $0.35{ }^{\circ} \mathrm{C}$.

In order to compare the temperature evolution at the same places, two areas $Z_{1}$ and $Z_{2}$ are defined. They locate at the same position on each sample. Figures $15 \mathrm{a}$ and $15 \mathrm{~b}$ 
(a). Sample without defect

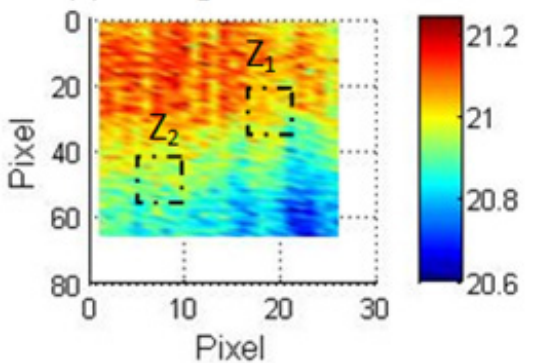

(c). Contrast of both samples

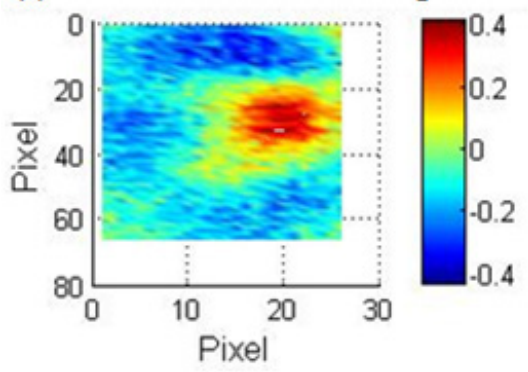

(b). Sample with defect

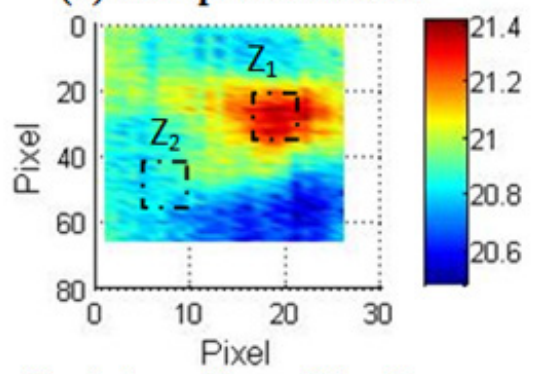

Position of metallic element

(d). Contrast compared to initial instant

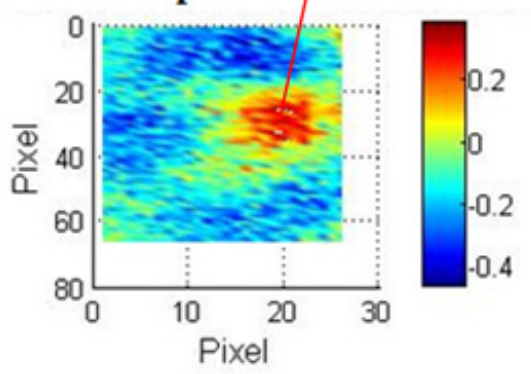

Fig. 14. Thermograms of the wooden plates at the instant of $170 \mathrm{~s}$.
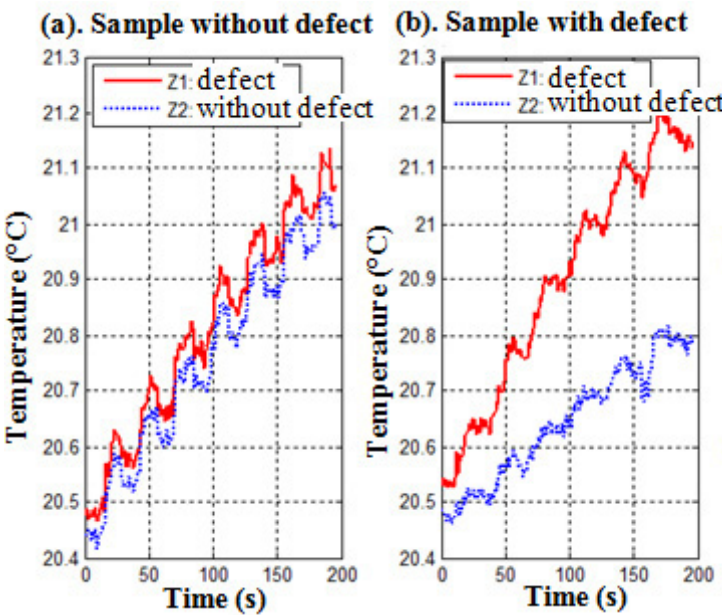

(c). Contrast between both samples

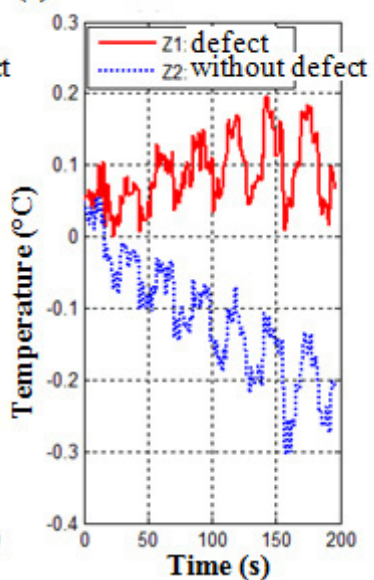

Fig. 15. Evolution of the surface temperatures of the wooden plates.

show the evolution of the temperature of the wooden plate samples with and without metallic element for both areas. Their development is modulated corresponding to the operating rhythm of the magnetron.

\section{Conclusion}

The microwave excitation applied to the carbon fiber reinforced polymer showed the accessibility to get the results similar to those obtained from conventional excitations (flash, halogen). The second series of the tests showed the interest of microwave excitation that enabled to reveal the presence of a metallic element behind a wooden plate of one centimeter of thickness. The effect of microwave excitation caused a moderated increase in temperature and does not lead to any alteration of detected materials. These preliminary works open interesting perspectives for inspecting the reinforced concrete structures.

\section{References}

[1] C.A. Balaras, A.A. Argiriou, Infrared thermography for building diagnostics, Energy Build. 34 (2002) 171-183

[2] C. Meola, A new approach for estimation of defects detection with infrared thermography, Mater. Lett. 61 (2007) 747-750

[3] Chia-Chi Cheng, Tao-Ming Cheng, Chih-Hung Chiang, Defect detection of concrete structures using both in- 
frared thermography and elastic waves, Automat. Constr. 18 (2008) 87-92

[4] E. Grinzato, V. Vavilov, T. Kauppinen, Quantitative infrared thermography in buildings, Energy Build. 29 (1998) 1-9

[5] F. Taillade, M. Quiertant, K. Benzarti, C. Aubagnac, Shearography and pulsed stimulated infrared thermography applied to a nondestructive evaluation of FRP strengthening systems bonded on concrete structures, Constr. Build. Mater. 25 (2011) 568-574

[6] H. Wiggenhauser, Active IR-applications in civil engineering, Infrared Phys. Technol. 43 (2002) 233-238

[7] Jeff R. Brown, H.R. Hamilton, Quantitative infrared thermography inspection for FRP applied to concrete using single pixel analysis, Constr. Build. Mater. (2010)

[8] M. Marchetti, S. Ludwig, J. Dumoulin, L. Ibos, A. Mazioud, Active Infrared Thermography for NonDestructive Control for Detection of Defects in Asphalt Pavements, presented at the 9th International Conference on Quantitative Infrared Thermography, Krakow, Poland, 2008

[9] M.R. Clark, D.M. McCann, M.C. Forde, Application of infrared thermography to the non-destructive testing of concrete and masonry bridges, NDT \& E Int. 36 (2003) 265-275

[10] Manyong Choi, Kisoo Kang, Jeonghak Park, Wontae Kim, Koungsuk Kim, Quantitative determination of a subsurface defect of reference specimen by lock-in infrared thermography, NDT \& E Int. 41 (2008) 119-124

[11] Maldague, Theory and Practice of Infrared Technology for Nondestructive Testing, Canada, 2001

[12] X. Maldague, Y. Largouët, J.-P. Couturier, A study of defect depth using neural networks in pulsed phase thermography: modelling, noise, experiments, Revue Générale de Thermique 37 (1998) 704-717
[13] D. Balageas, J.-C. Krapez, L. Legrandjacques, F. Lepoutre, H. Petry, Industrial applications of infrared thermography, presented at the 10th International Conference, CP463, Photoacoustic and Photothermal Phenomena, Roma Italy, 1998

[14] U. Galietti, D. Palumbo, G. Calia, F. Ancona, New data analysis to evaluate defects in composite materials using microwaves thermography, presented at the 11th International Conference on Quantitative InfraRed Thermography, University of Naples Federico II, Naples, Italy, 2012

[15] G. Cuccurullo, P.G. Berardi, R. Carfagna, V. Pierro, IR temperature measurements in microwave heating, Infrared Phys. Technol. 43 (2002) 145-150

[16] G. Cuccurullo, V. Pierro, A procedure to measure electromagnetic skin depth in microwave heating, Infrared Phys. Technol. 46 (2004) 49-55

[17] K. Güney, Simple Design Method for Optimum Gain Pyramidal Horns, AEU - Int. J. Electron. Commun. 55 (2001) 205-208

[18] R. Kitchen, RF and Microwave Radiation Safety Handbook, 2nd edition, Oxford, 2001

[19] J.L. Pedreño-Molina, J. Monzó-Cabrera, M. Pinzolas, A new procedure for power efficiency optimization in microwave ovens based on thermographic measurements and load location search, Int. Commun. Heat Mass Transfer 34 (2007) 564-569

[20] Juan Monzó-Cabrera, J.L. Pedreño-Molina, A. Toledo, Feedback control procedure for energy efficiency optimization of microwave-heating ovens, Measurement 42 (2009) 1257-1262

[21] M. Bangay, C. Zombolas, Advanced Measurements of Microwave Oven Leakage, presented at the Australian Radiation Protection and Nuclear Safety Agency, Australia, 2004 